\title{
Geben Ärzte bei Demenz zu oft Antipsychotika?
}

\author{
Hausärzte verschreiben bei Demenz seltener Antidementiva als Neurologen und Psychiater. \\ Antipsychotika werden dagegen von beiden Gruppen erstaunlich oft verordnet.
}

_ Seit vielen Jahren werden in Europa und in den USA Abweichungen von den Therapieleitlinien bei Demenz beobachtet. Der Schluss liegt nahe, dass zu wenig Antidementiva und zu viel Antipsychotika verordnet werden.

Für eine deutsche Studie wurden nun Daten der GKV von 2011 analysiert. Dabei flossen die Verschreibungszahlen für beide Medikamentengruppen sowie die demenzspezifischen ICD-10-Diagnosen ein. Haus- und Fachärzte wurden getrennt betrachtet. Insgesamt wurden 1.014.710 gesetzlich Versicherte unter der Diagnose einer Demenzerkrankung behandelt. 45\% der Demenzdiagnosen waren nicht weiter spezifiziert.

Ein Antidementivum erhielten 24,6\% aller Patienten mit einer Demenzerkrankung. Wurde spezifisch eine AlzheimerDemenz diagnostiziert, stieg der Anteil auf $42 \%$. Die Wahrscheinlichkeit, mit einem Antidementivum behandelt $\mathrm{zu}$ werden, verdoppelte sich, wenn ein Neurologe oder Psychiater in die Behandlung involviert war.

Ein Antipsychotikum erhielten 35\% aller Demenzpatienten. War nur der Facharzt an der Behandlung beteiligt, war eine solche Medikation nicht wesentlich seltener (Odds Ratio: 0,86).

Die Autoren schließen daraus, dass durch den Einbezug eines Neurologen oder eines Psychiaters leitliniengerecht mehr Antidementiva zum Einsatz kommen. Der Einsatz von Antipsychotika zeigte sich aber im Gegensatz zu den Leitlinien bei allen Arztkonstellationen als überraschend hoch.

- Bohlken J, Schulz M, Rapp MA et al. Pharmacotherapy of dementia in Germany: Results from a nationwide claims database. Eur Neuropsychopharmacol 2015:25:2333-8

\section{KOMMENTAR}

Dass bei über einer Million Menschen in Deutschland 2011 die Diagnose einer Demenzerkrankung vermerkt wurde, zeigt, dass die Demenz nicht mehr als "normale" Altersschwäche verkannt, sondern als Krankheit dokumentiert wird. Fachärzte neigen eher als Hausärzte zur Vergabe von spezifischen Demenzdiagnosen und zum Einsatz von Antidementiva. Ein Besuch beim Facharzt zur Sicherung der Diagnose und der Einleitung einer spezifischen Arzneimitteltherapie sollte in unserem Gesundheitssystem Standard sein.

Die hohen Verordnungszahlen für Antipsychotika werfen die Frage auf, ob es im Einzelfall keine nicht-medikamentösen Vorgehensweisen gäbe oder ob diese im Praxisalltag nicht wirken. Hier besteht ein hoher, versorgungsrelevanter Forschungsbedarf.

Prof. Dr. med. M. Hüll

\section{So extrem kann Gicht aus dem Ruder laufen}

Ein 59-jähriger Mann wurde wegen multipler Knoten an beiden Händen in eine Universitätsklinik in Shanghai überwiesen. Vor längerer Zeit war bei inm durch den Nachweis doppelbrechender Kristalle im Gelenkpunktat eine Gicht-Arthritis diagnostiziert wurden. Er hatte unregelmäßig Allopurinol und Benzbromaron eingenommen. Zusätzlich waren eine Hypertonie und ein Diabetes mellitus bekannt. Der Mann wies multiple Gichtknoten an den Ohrmuscheln, im Bereich von Knie-, Sprung-, Finger- und Zehengelenken auf. Besonders ausgeprägt waren die Befunde an seinen Händen (Abb. A, B), wo es bereits zu einem schweren Funktionsverlust der Gelenke gekommen war. Die Serumanalyse ergab einen Kreatininwert von $1,6 \mathrm{mg} / \mathrm{dl}$ und einen Harnsäurewert von $12,2 \mathrm{mg} / \mathrm{dl}$.

Unter der Diagnose einer fortgeschrittenen erosiven tophösen Gicht wurde er zunächst mit Febuxostat behandelt. Nach einem Monat war die Harnsäurekonzentration auf 9,7 mg/dl gefallen. Offenbar hatte sich der Mann an seinen Zustand gewöhnt, da er sich weiteren Kontrollen und einer möglichen chirurgischen Behandlung entzog.

Gichttophi entstehen in der Regel erst nach längerer Zeit, in der Regel nach etwa zehn Jahren. Die Autoren konstatieren, dass Tophi an den Händen selten seien. Vielleicht ist das in China so, in Europa sind sie aber nicht ungewöhnlich, wie ich aus eigener langjähriger Erfahrung in einer Stoffwechselambulanz weiß. Auch scheinen die Ansprüche in China bei der Gichttherapie nicht besonders hoch zu sein. Mit einem Idealwert von $6 \mathrm{mg} / \mathrm{dl}$ würde man sich hierzulande nicht zufrieden geben. Derart fortgeschrittene Befunde erfordern auch eine chirurgische Intervention, schon um die Niere zu entlasten, die diese Unmengen von Urat ja schließlich ausscheiden muss.

Prof. Dr. med. H. S. FüeßI
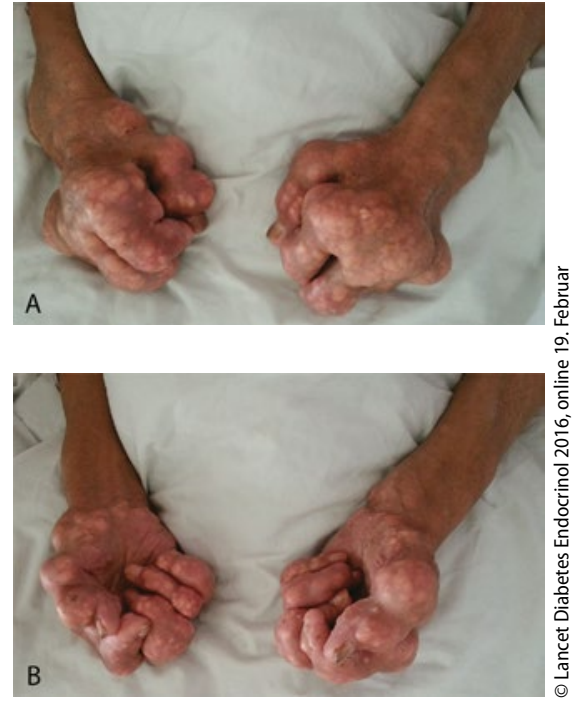

Multiple, behindernde Gichtknoten an beiden Händen.

- Han B et al. (yangchengde@sina.com). Giant gouty tophi in the hands. Lancet Diabetes Endocrinol 2016, online 19. Februar 